\title{
A Hardware and Software Framework for Automotive Intelligent Lighting
}

\author{
Marko Heiko Hörter and Christoph Stiller \\ Institut für Mess- und Regelungstechnik \\ Karlsruhe Institute of Technology (KIT) \\ D-76131 Karlsruhe, Germany \\ Email: \{hoerter, stiller\}@mrt.uka.de
}

\author{
Christian Koelen \\ Advanced Driver Assistance Systems \\ Porsche Engineering Services $\mathrm{GmbH}$ \\ D-74321 Bietigheim-Bissingen, Germany
}

Email: christian.koelen@porsche-engineering.de

\begin{abstract}
This paper describes the lately completed hardware and software framework in the field of automotive intelligent lighting. Furthermore a brief overview of currently announced concepts within this focus as well as a deducted tendency will be given. As marking spot lights will draw the driver's attention to hazardous objects in the future, the "Automotive Spot Light" as a novel approach on this will be announced. The combination of visual as well as thermal imager both with their advantages will help to decrease fatal injury on the road plus enables new safety light applications.
\end{abstract}

\section{INTRODUCTION}

An accident analysis recently performed by the German federal statistical office (DESTATIS ${ }^{1}$ ) points out the number of persons who have been killed in the year 2008. The result represents an all-time low since the collecting of those data have been started in 1953. Nevertheless the absolute number of approximately 4.600 persons killed as well as 407.000 persons injured [1] gives obviously sufficient motivation for all related institutions (particularly governmental, scientific research, and industrial institutions) to keep on working together with the common intention to reduce personal injury within the road traffic context.

Especially at night time when visual perception for drivers is at the lowest level, the risk of accidentally collision between vehicles and other objects (here: focussing on human beings, animals, etc.) increases disproportionately high. Less ambient light, artificial automotive lighting, lower contrasts and reduced visibility are just some reasons at this.

A database extract of [2] provides clear figures concerning accident frequency as well as main accident types investigated by specialist teams of GIDAS ${ }^{2}$. In Figure 1 can be seen the accident types "(1) Loss-of-control", "(2) Turning off the Road", and "(3) Crossing a road" show a significant relative increase at night making them high potentials regarding further analytical consideration.

More detailed (1) are representing mainly so called breakaway accidents, which often result from maladjusted driving speed based on incorrect perception due to moderate illumination of the road border area. (2) are quite typical accidents during night time as well, in which vehicle collide at

\footnotetext{
${ }^{1}$ http://www.destatis.de

${ }^{2} \mathrm{http}: / /$ www.gidas.org
}

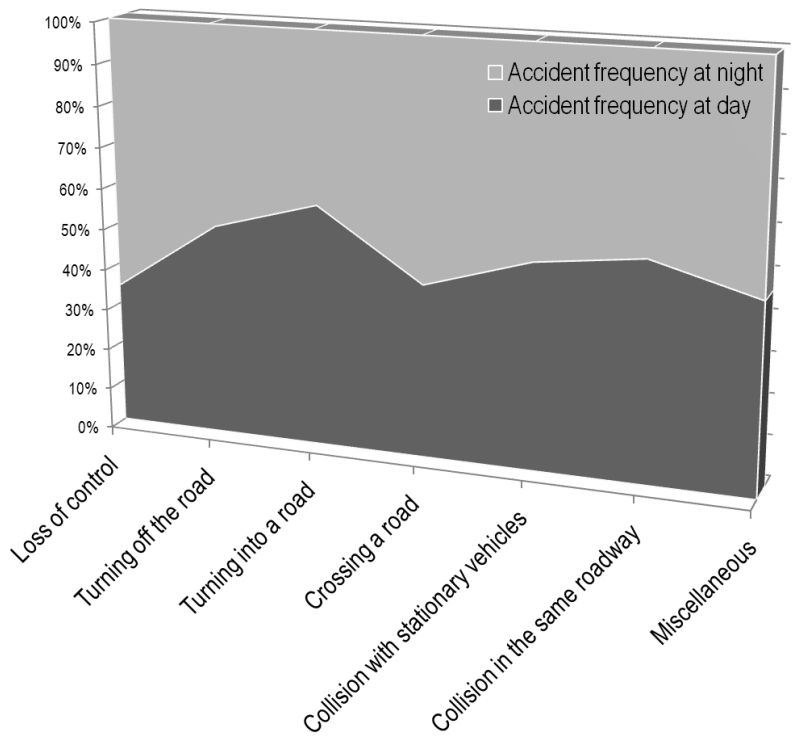

Fig. 1. Accident frequency at day and night time [3]

intersection area (mostly during a left turn). In given accident reports the person who has caused the accident stated not to have seen the oncoming vehicle due to a lack of illumination. (3) represent amongst others mainly pedestrian accidents, in which the driver does not have enough or even no time to react properly to pedestrians or animals often unexpectedly crossing the road[3].

Those identified main accident types are setting up the framework for further automotive intelligent lighting development to bring the above stated numbers of killed or injured people down further.

Several car manufacturer (OEM) as well as system supplier are currently researching how to support the driver best during night time on a technical perspective. Hereunto the paper's second section gives an up-to-date overview about recently announced systems within this focus. Followed by the third section describing a novel system architecture, which is designed to evaluate multiple algorithm in the field of intelligent lighting. With special focus on the so called "Marker Light" (introduced in [4]), the research and testing platform features 
a prototype headlight system, a real-time database as well as a real-time controller framework. The fourth section gives an outlook on the research topics based on the introduced platform which are designated in the future.

\section{General OVERVIEW INTELLIGENT Lighting}

The combination of lately announced developments in the field of headlight technology and (here: visual) environmental detection enables new safety light application. This section gives a rough overview about recently introduced intelligent lighting (or supportive) systems split up by car manufacturer and system supplier with the common aim to increase the driver's perception and herefrom his safety at night time.

\section{A. Car Manufacturer (OEM)}

1) BMW Group: ${ }^{3}$ By launching the latest BMW 7 th series to the market a wide variety of light-based driver assistance systems has been introduced. In [5] the so called "Dynamic Headlight Range Adjustment" system controls the leveling of the xenon headlights due to the vehicle's payload (delayedaction leveling) as well as corresponding to the vehicle's acceleration (dynamic and non-delayed-action leveling). By calculating the derivation of the vertical curvature linked to the current route section an implemented algorithm is capable to predict the further vertical road topology and perform hereby a semi-predictive headlight leveling functionality based only on vehicle immanent data.

Likewise based only on internal vehicle information the "Adaptive Cornering Light" comprises a static as well as a dynamic horizontal light distribution. Here the horizontal curvature is calculated by the vehicle's yaw rate and steering angle as input. As an enhancement on this the "Adaptive Light Distribution" functionality controls, based on optical sensor information, the horizontal light distribution of the vehicle's headlamp corresponding to the given environment. The following lighting pattern will be chosen according to the implemented algorithm automatically:

Motor highway: Specialized for long range visibility.

Cross country road: Compromises mid range visibility and mid flat illumination.

Urban environment: Flat illumination, specialized for best recognition of pedestrian and bicyclist.

Adverse weather: Designed for low distance as well as flat illumination, hereby low reflection due to snowy or wet roads.

The so called "High Beam Assistant" uses the same multipurpose camera system. Here an automatic alternation between low and high beams is carried out based on a traffic situation evaluation, which has detected, tracked, and finally classified valid lighting blobs in the image data.

The BMW's FIR-'Night Vision" system in its second generation features an announced pedestrian detection functionality. BMW states an enhanced visibility up to $300 \mathrm{~m}$ by using the "Central Information Display" (CID). The driver is getting

\footnotetext{
${ }^{3}$ http://www.bmw.de
}

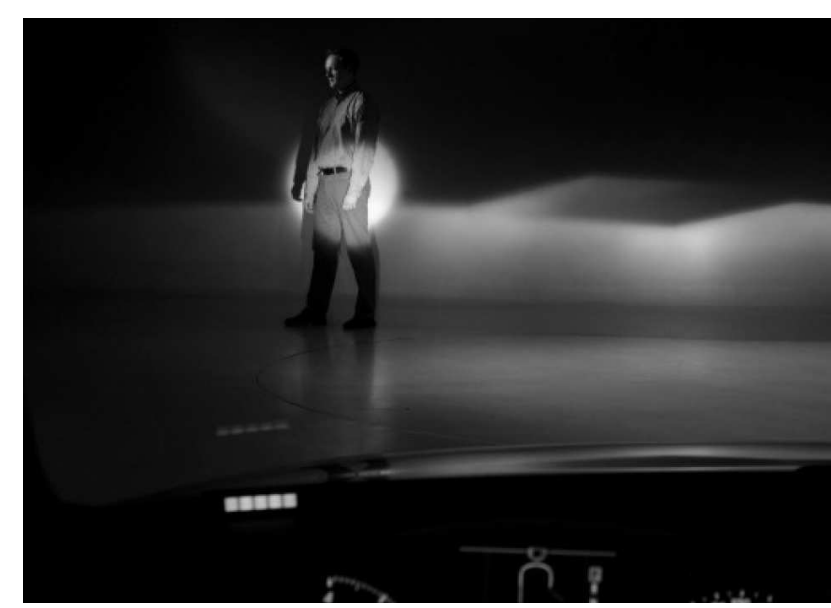

Fig. 2. Example picture of Volkswagen“s "Marking Light"[3]

visual warnings through the CID as well as displayed on the Head-up-display (HUD). [6] states the prime beneficial usage during nighttime on cross country roads, due to the statistically highest risk of accidentally collision with pedestrian. The classification algorithm is done by a Support-VectorMachine (SVM), which has gotten a training set database of thousands of international environmental pictures (pedestrians, bicyclists).

2) Volkswagen AG: 4 The Volkswagen AG has recently published its so called "Active Safety Light" concept. It is a combination of new headlight technologies and environment detection based on automotive imager. [3] says, that it is possible to draw driver's attention with the "Active Safety Light" to potential hazardous areas within the road traffic context. The presented approach of the technical system is based on a carried out detailed accident evaluation. Some advanced lighting functionalities, like the "Adaptive Front Lighting System (AFS)" (statical and dynamical bending lights coupled to vehicle parameter like steering angle and velocity) as well as the "Adaptive Cut-Off-Light" (using the visual sensor data to observe the traffic ahead and adapt the light range automatically) were described. In addition the so called "Masked High Beam" system is using the visual sensor data as well to observe the traffic ahead to dim selected areas where other road user could be blinded. As a result on that the driver should be able to observe traffic much more detailed at night time plus is capable to react earlier in dangerous situation. A sub-project named "Marking Light" is designed to assist ever when objects suddenly appear (e.g. pedestrian who surprisedly walks on the road) or if the driver is distracted by other light sources. Hereby a generated light spot will redirect the driver's attention in direction of e.g. crossing pedestrians (see Figure 2). On the sensor side Volkswagen has denoted to utilize a night vision system - to perform the focused light spot variable light actuators will be needed, which could be realized by a "Matrix-Beam headlamp".

\footnotetext{
${ }^{4}$ http://www.volkswagen.de
} 


\section{B. System Supplier}

1) Hella KGaA Hueck \& Co: ${ }^{5}$ In [4] Hella has announced a wide spectrum of camera-controlled "Light Based Driver Assistance Systems" from the system supplier perspective. The following systems were introduced:

"Full adaptive front lighting systems (FAFS)": In contrast to VW's AFS system, Hella has combined vehicle immanent data (e.g. velocity, steering angle, etc.) with visual data provided by an automotive imager (here: CMOS imager). Hereby the light distribution adapts automatically to speed, street as well as to weather and traffic conditions on the road.

"Adaptive cut-off line": The idea behind "Adaptive cut-off line" is to have the low beam not to stop around $65 \mathrm{~m}$, as it was usually the case, but rather increase the visual range for the driver to a maximum laterally not to dazzle other road user. A detection of any traffic participant at night up to a distance of $800 \mathrm{~m}$ is necessary, so Hella. The adaptive cut-off line is featured by the so called "Hella VarioX" xenon projection system.

"Glare-free high beam":

For the "Glare-free high beam" system, the light emitted by the headlamps is only directed into those regions, in which no glare for other traffic participants could be created. In other words the driver travels almost the whole time with permanently switched on high beams, except of faded out areas where other road user appear. Hella is currently investigating two promising technologies for the realization of those dynamic light functions: Either the so called "VarioX" xenon projection system featuring a special contoured rotatable drum to generate continues faded out areas or an LED-array headlamp, which does not need moving elements to create different lighting distribution, could be utilized.

"Marker Light":

The exactly opposite manner to the "Glare-free high beam" represents the so called "Marker Light". The goal hereby is to draw the driver's attention as early as possible to dangerous objects within the traffic context. To follow the hazardous object with the marker light, an adaptive light source based on LED-arrays is currently under development by Hella.

2) Autoliv Electronics $A B:{ }^{6}$ Autoliv describes in [7] a state-of-the-art night vision system approach, which performs pedestrian detection in the darkness. The system uses a FarInfrared (FIR) camera which requires no active illumination. The system features basically three main modules: Image enhancement, pedestrian detection and tracking, as well as a warning strategy. Autoliv states by using the presented system the driver's range of sight will increase from approximately $40 \mathrm{~m}$ to around $90 \mathrm{~m}$ at night.

\footnotetext{
${ }^{5}$ http://www.hella.de

${ }^{6} \mathrm{http}: / / \mathrm{www}$.autoliv.com
}

The purpose of the image enhancement module is to give the driver an optimized visual representation of the original captured video data due to a lack of visual quality. To remove noise, a noise reduction step represented by a low-pass filter algorithm is carried out. Followed by an edge enhancement, grey-level stabilization as well as a final histogram manipulation algorithm the visual representation of the thermal image increases.

The initial step of the pedestrian detection module is to extract potential candidates - followed by the classification process, which discriminates between pedestrian and other objects. A final tracking algorithm of the classified pedestrians estimates the relative position and velocity, which are used in the following warning strategy module.

In order to focus the driver's attention only on risky areas, a warning strategy module needs to be implemented. Inside this module, a decision making process is carried out, which evaluates if a warning is to be issued towards the driver or not. Hereby an adaptive warning range corresponding to the calculated time-to-impact (TTI) has been described.

As a visual sensor, Autoliv uses a passive un-cooled bolometer FIR system, which is working on a 8-12 $\mu \mathrm{m}$ wavelength base. That specific physical range has been chosen due to the fact the maximum thermal energy is emitted here by warm-blooded beings.

3) Valeo Lighting Systems: ${ }^{7}$ Among the currently available lighting system called "Intelligent Lighting", which features a dynamic bending light, Valeo has lately announced in [8] an approach how to combine lighting automation and advanced lighting units to achieve a continuous transition between low and high beam illumination. The system includes a multifunction RGB CMOS imager as well as image processing which analyzes the road environment and detects presence and relative position of other vehicles. As an output of the image processing unit the relative vertical position of detected vehicles to control headlamp leveling as well as the estimated distance to other vehicles due to range adjustment of the xenon lighting unit were described.

The system supplier introduces its product "BeamAtic Plus", which controls the beam position in three dimensions - the spatial position is hereby defined by the distance to the target plus the vertical and horizontal angle. The algorithm on this consists firstly of a lamps detection to be due to pattern recognition and gradient recognition as well as a final thresholding. The second step integrates a tracking algorithm followed by a trajectories identification to find valid and reject non-valid objects. The described distance estimation is based on kalman filtering, which estimates, predicts, and smoothes the distance evaluation. Beside the estimated longitudinal distance the lateral distance is defined by measuring the pixel gap in the camera reference.

Valeo introduces contemporaneously a progressive beam module named "Trixenon", which allows continuous movement of the internal light shield by a rotatable drum. By

\footnotetext{
${ }^{7} \mathrm{http}: / /$ www.valeo.com
} 
moving down the light shield, the total quantity of lumen plus maximum light intensity increases as well as the cut-off line is moving upwards.

\section{System ARChitecture}

By summing up the previous section it is getting obvious, that both car manufacturer as well as system supplier currently researching on the following four main topics in the field of intelligent lighting systems:

- (Full) Adaptive front lighting system,

- Adaptive cut-off line,

- Glare-free high beam,

- Marking light.

As the different described technical solutions pointing out, a comprehensive system architecture in the field of intelligent lighting should compromise the following technical items:

Automotive Imager:

An automotive imager should be utilized to capture visual data in front of the ego-vehicle with respect to adequate resolution and frame rate.

Far-Infrared (FIR) Imager: To extend the driver's visibility on a technical perspective at nighttime, an FIR system provides important information.

Advanced headlight module: To have the cut-off line perform continuous transition corresponding to the traffic situation, on the one hand side with respect to xenon projection systems a special contoured instance (e.g. rotatable drum) should be integrated. On the other hand side with respect to LED-array modules the light performance has to be guaranteed.

Marking Light:

To direct the driver's attention to identified hazardous objects within the traffic context, a high-dynamic lighting instance should be implemented.

Advanced Software Modules: Image enhancement, detection, and tracking of potential objects as well as a final classification should be mandatory.

The following sub-sections describe a recently accomplished hard- and software framework at our research department, which fulfills the above listed requirements to be capable to carry out further research in the field of automotive intelligent lighting.

\section{A. Hardware components}

1) Vehicle platform: As a testing platform at our department an AUDI Q7 3.0 TDI vehicle is available and ready to use. Since the technical framework developed at our as well as at our partners' department described in [10] has shown great performance and reliability, a similar basic setup has been integrated in the AUDI.

2) Prototype head lamp including the "Automotive Spot Light": Figure 3 shows the lighting system which was lately completed. To meet the above stated requirements, two sub-modules were adapted/ developed to fit into the seriesproduction head lamp body:

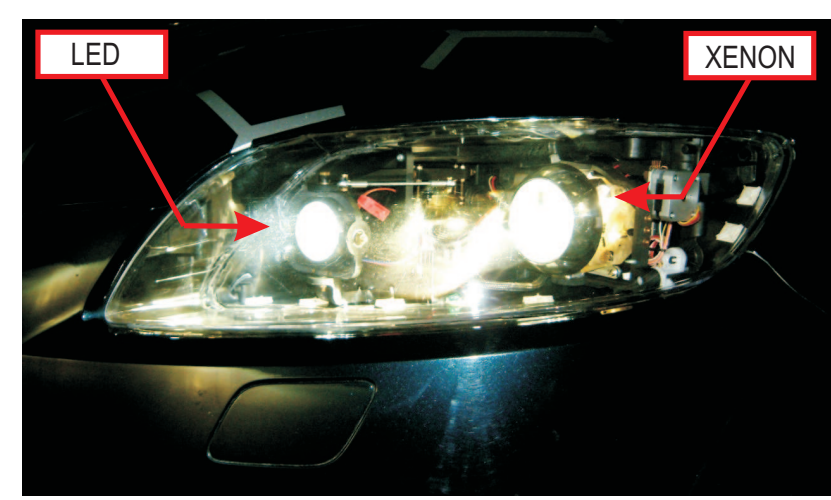

Fig. 3. Integrated prototype head lamp system

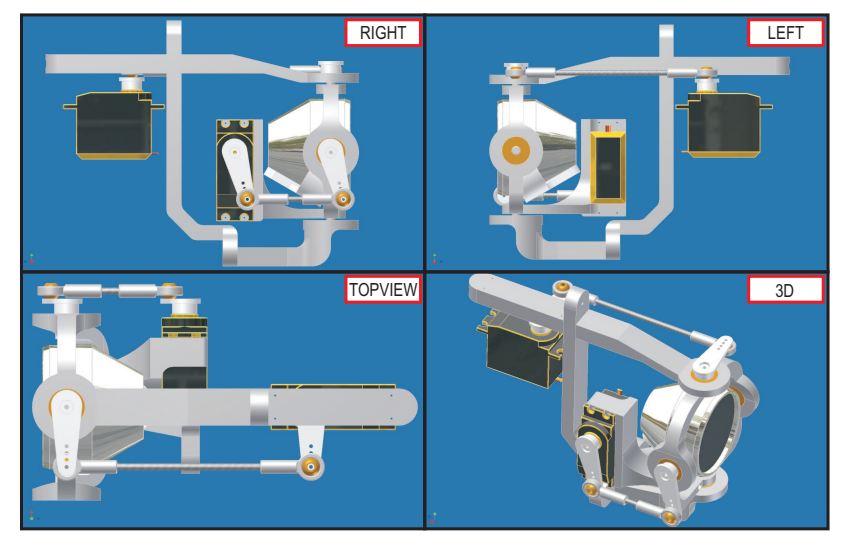

Fig. 4. 3D-CAD drawing "Automotive LED Spot Light"

- Advanced xenon module with continuous adjustable light shield (e.g. rotatable drum),

- Prototype "Automotive LED Spot Light".

Hereby the xenon sub-module consists of the following items:

- Xenon projection system,

- Actuator to yaw the projection system,

- Actuator to pitch the projection system,

- Actuator to adjust (rotate) the light shield,

- Electronic Control Unit (ECU).

The prototype "Automotive LED Spot Light" sub-module (Figure 4) includes the following elements:

- High power multi-core LED emitter with > 1000 lumen of light power,

- Optical system to perform focused light spot on hazardous objects with adequate luminous density,

- Actuator to yaw the LED system,

- Actuator to pitch the LED system.

The communication to the xenon sub-module is done by CAN communication protocol via ECU front end - the control of the installed actuators is realized by the ECU back end. The electronic connectivity towards the actuators of the second sub-module is done by the later on specified $\mathrm{dSPACE}^{8}$ tool

\footnotetext{
${ }^{8} \mathrm{http}: / /$ www.dspace.de
} 
chain based on amplified pulse-width modulation (PWM) signals. To adjust the position of the two actuators (servo drive), the duty cycle of the constant frequency PWM signal has to be controlled. To dim the high power multi-core LED emitter on a linear scale form zero to maximum correlating to an analog input signal, a MOS-FET driver circuit has been developed.

3) Computing system hardware: The on-board computing system hosts all software for perception and decision making. It has to provide sufficient computing power and has to guarantee low latencies for interprocess communication. To fulfill these requirements a multicore multiprocessor computer system using opteron processors has been chosen. All opteron processors are interconnected by HyperTransport (HT) at a speed of 3,2 GByte/s each. For logging purposes a very fast raptor hard disk is used, providing $44-91 \mathrm{MB} / \mathrm{s}$ and sustaining accelerations up to $3 g[10]$.

4) Visual sensors: As visual CMOS sensor a PointGrey ${ }^{9}$ "Firefly MV" module has been chosen to grab images during night time. To avoid motion blur within the visual near field at night, an adequate frame rate of $\geq 30 \mathrm{fps}$ with a resolution of $640 x 480$ pixel has been set. Reflections, which will occur e.g. from wet road surface, can be smoothen by using a polarizing filter.

Concerning the acquisition of thermal images the FLIR ${ }^{10}$ "PathFindIR" unit have been integrated into the framework. With a particularly designed housing for automotive application, a resolution of $320 \times 240$ uncooled microbolometer and a frame rate of approximately $25 \mathrm{~Hz}$ is the unit linked via ethernet to the computing system.

5) Real-time microcontroller: To guarantee real-time control capability, a dSPACE "MicroAUTOBOX" as well as a dSPACE "RapidPro Unit" were integrated in the system architecture. Those tools make it possible, to control all the above listed actuators electronically as well as flash down complex MATLAB/ Simulink ${ }^{11}$ models to the target system. dSPACE's "MicroAUTOBOX" (here: 1401/1505/1507) provides reliable interface capabilities (ADC, DAC, DIO, CAN, [FleyRay], etc.) - the linked "RapidPro Unit" converts TTL leveled signals into specified power signals.

6) Inertial Measurement Unit (IMU): As precise positioning tool the OXTS 12 "RT3003" Inertial and GPS Navigation System with six-axis inertial navigation strap down system is used. It incorporates an L1/L2 RTK GPS receiver for position and a second GPS reveiver for accurate heading measurement[10].

7) Power unit: In order to operate the computing system independently of the vehicle's battery, a power unit was installed. The power unit compromises two gel accumulator, $\mathrm{DC} / \mathrm{AC}$ and $\mathrm{DC} / \mathrm{DC}$ converters, a charging device, fuses, and buffer condensers.

\footnotetext{
${ }^{9}$ http://www.ptgrey.com

${ }^{10} \mathrm{http} / / / \mathrm{www}$. flirthermography.de/

${ }^{11}$ http://www.themathworks.com

${ }^{12} \mathrm{http}: / /$ www.oxts.co.uk
}

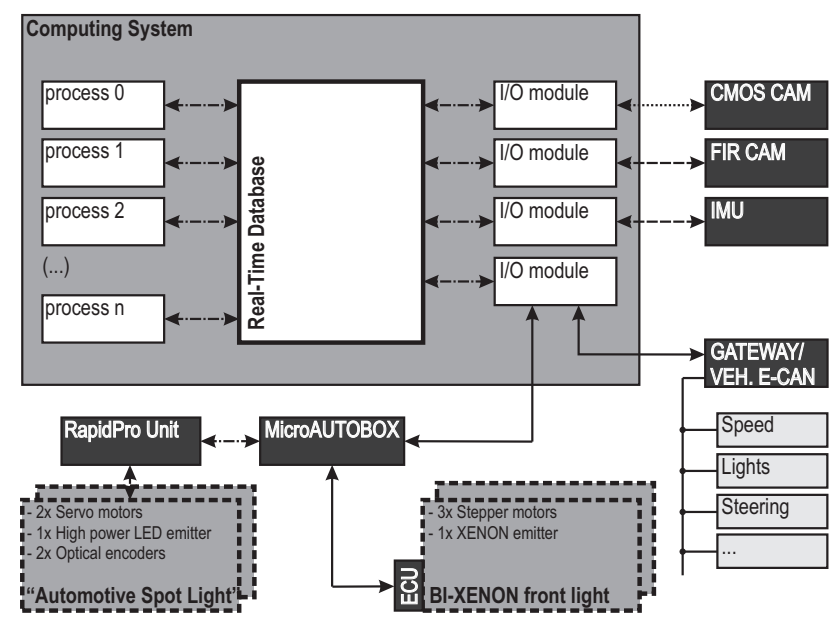

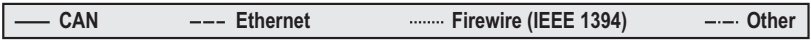

Fig. 5. System architecture and information flow diagram

\section{B. Data Transmission}

Figure 5 gives an survey of the information flow between all connected sensor-, computing-, and, actuator instances. The FIR and IMU sensors are linked to the central computing system with a $100 \mathrm{MBit} / \mathrm{s}$ ethernet connection. The received raw data is stored into the RTDB through the I/O modules and is being processed. The "MicroAUTOBOX" receives and sends its information mainly via high-speed CAN at $500 \mathrm{KBit} / \mathrm{s}$. The connected "RapidPro Unit" receives data via TTL level and concert them into amplified analog/ digital output data. The CAN I/O module installed within the computing system serves two CAN channels, one for the "MicroAUTOBOX", the second to (only) receive data from the vehicle E-CAN bus. CMOS camera data is streamed via firewire.

\section{Software components}

1) Real-time database: An implemented real-time database (RTDB, [9]) acts as the central communication module for high-level decision making on the computing system. Every process is connected only to the RTDB and to no other process. The interprocess communication is applied conform the following methods:

- All processes have at least one logical database connection.

- Every set of data is packed together into an "object", the smallest unit handled by the RTDB.

- The sending process inserts an object with the relevant data into the RTDB.

- The receiving process uses the RTDB-API to find the object and retrieve its data.

- Whenever the sender has new data available, it immediately updates the object.

- The receiver waits for new data and is informed as soon as it is submitted. 
- The data is automatically buffered within the RTDB for an adjustable time interval, so it is possible to retrieve older data, e.g. to combine video data with the correct GPS-position at the time the measurement has been performed.

Due to the efficient implementation of the real-time database, it takes only $8.3 \mu \mathrm{s}$ to update an object and $6.4 \mu \mathrm{s}$ to retrieve it. The full communication from one process to another takes $29.6 \mu s[10]$.

2) Real-time controller framework: Since DSPACE provides Matlab/ SIMULINK blocksets, the I/O assignment of the actuators connected to controller box is straightforward. After setting up the SIMULINK model with all port assignments, the tool "Real-Time Workshop" is carried out to build the model by meaning of converting it into compilable and linkable instances as well as finally flash it down on the target system. After successfully flashing down the model, the controller box runs self-sufficient.

\section{Future WORK}

Due to the presented hardware and software framework incorporating all necessary requirements (or even more) to research in the field of automotive intelligent lighting, no major changes are scheduled on that in the future. As intended our focus will be on the following topics:

Enhanced software modules: Beside the important basic software modules introduced in this paper to ensure information flow and processing (e.g. RTDB) it will be as well as important to have reliable and precise software for detection, tracking, and, classification purposes handy. If there are some further improvements possible referring to the introduced software modules in the second section has to be investigated. Concerning the usage of enhanced FIR-based classification methodes (e.g. SVM), different "appearancebased" as well as "model-based" training set data have to be found[11].

Operation strategy: As automotive lighting have to be reliable and not to cause dazzling of other road user, novel operation strategies are required. To get the best performance out of the overall system, data fusion of both CMOS and FIR sensor will be mandatory. Hereby the CMOS information (cf. range up to $800 \mathrm{~m}$ ) could work as a so called global enabler, which reliably provides the spatial position of each illuminant. The FIR information, which triggers e.g. the marking lights, have to be validated first not to thwart other road user. Beside the safety features the as many as important manmachine interaction aspects (e.g. ergonomics) on this have to be evaluated, too. For instance the dimming strategy could be termed.

Validation tool: $\quad$ The spatial position of the detected and tracked illuminants has superior importance for many instances followed up. Monocular as well as stereo vision systems are currently under development. Therefore software simulation plus hardware-based validation tools will be seen in the future.

\section{CONCLUSION}

The carried out extensive literature research termed in the second section shows clear tendencies concerning the discussed field of research to have at least four main intelligent lighting functionalities in future vehicles. The proposed hardware and software framework has been implemented in the vehicle platform and its basic software modules showed reliable performance in various validation tests. The system will host enhanced software modules to detect, track and classify objects both in the visible as well as far-infrared light spectrum in the near future. To estimate the spatial distance of objects of interest will have prior significance. How the gained visual and thermal information could be merged together to generate the best ergonomic results for the driver during night time, is being under current research. With focus on the "Automotive Spot Light" instance, a novel dynamic lighting source has been presented to develop and evaluate real-time control strategies regarding marking light - hereby detached of currently ongoing LED-array head lamp developments.

\section{ACKNOWLEDGMENT}

This work is supported by Porsche Engineering Services $\mathrm{GmbH}$ as well as partly by the Collaborative Research Center (SHB/ Tr28) "Cognitive Automobiles" granted by the Deutsche Forschungsgemeinschaft.

\section{REFERENCES}

[1] Statistische Bundesamt, Zahl der Verkehrstoten: Neuer Tiefststand im Jahr 2008, Pressemitteilung Nr.479 vom 12.12.2008., Germany: Wiesbaden, 2008.

[2] German In-Depth Acciendet Study, GIDAS database, www.gidas.org, 2008.

[3] Christian Wegwerth, et al., Active Safety Light, V.I.S.I.O.N proceedings ref 2008-02, France: Versailles Satory, 2008.

[4] Christian Amsel, et al., Light Based Driver Assistance System, V.I.S.I.O.N proceedings ref 2008-02, France: Versailles Satory, 2008.

[5] Tobias Strobel, et al., Licht intelligent umgesetzt, Elektronik automotive - Sonderausgabe BMW 7er, Germany: Poing, 2008.

[6] Sebastian Orecher, et al., Souvern und sicher - auch bei Nacht, Elektronik automotive - Sonderausgabe BMW 7er, Germany: Poing, 2008.

[7] Qingfen Lin, et al., A Far-Infrared Based Night-Vision System with Pedestrian Detection, V.I.S.I.O.N proceedings ref 2008-02, France: Versailles Satory, 2008.

[8] Julien Moizard, et al., Advanced High Beam/ Low Beam Transitions: Progressiv Beam And Predicitve Leveling, V.I.S.I.O.N proceedings ref 2008-02, France: Versailles Satory, 2008.

[9] Matthias Goebl, et al., A Real-Time-capable Hard-and Software Architecture for Joint Image and Knowledge Processing in Cognitive Automobiles, In Proc. IEEE Intelligent Vehicles Symposium, pages 734-740, Turkey: Istanbul, 2007

[10] Moritz Werling, et al., A Hardware and Software Framework for Cognitive Automobiles, IEEE Intelligent Vehicle Sympossium, The Netherlands: Eindhoven, 2008.

[11] Dariu M. Gavrila, Looking at People, 38. Heidelberger Bildverarbeitungsforum: Menschen und Bildverarbeitung, Germany: Heidelberg, 2008. 